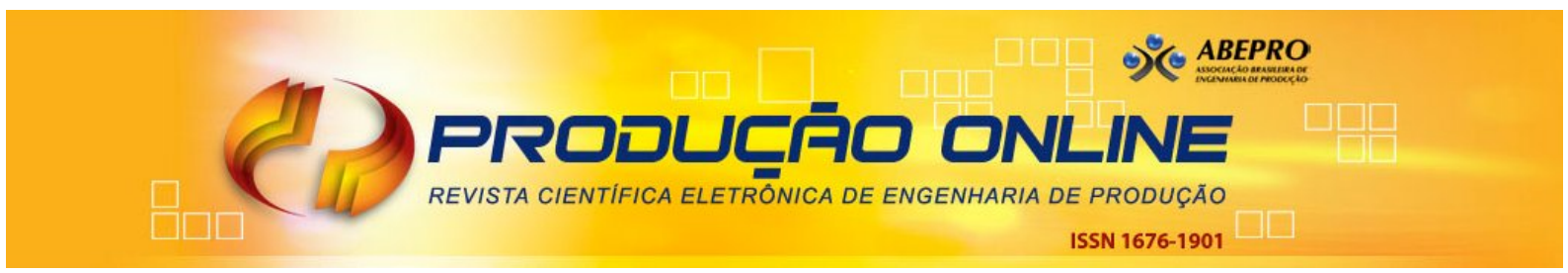

\title{
CONTRIBUIÇÃO DO MÉTODO SISTEMA ESPECIALISTA FUZZY NA ANÁLISE DE IMPACTO REGULATÓRIO
}

\section{CONTRIBUTION OF A FUZZY EXPERT SYSTEM TO REGULATORY IMPACT ANALYSIS}

\author{
Marco Antônio da Cunha Ferreira* E-mail: macfe@ele.puc-rio.br \\ Alessandro Copetti^* E-mail: alessandro.copetti@gmail.com \\ Alex da Silva Alves ${ }^{\star \star \star}$ E-mail: alexds.alves@gmail.com \\ Carlos Alberto Malcher Bastos ${ }^{\star \star \star \star}$ E-mail: cmbastos@telecom.uff.br \\ * Pontifícia Universidade Católica do Rio de Janeiro (PUC-RJ), Rio de Janeiro, RJ \\ ** Universidade Federal Fluminense (UFF), Rio das Ostras, RJ \\ *** Universidade de São Paulo (USP), Piracicaba, SP

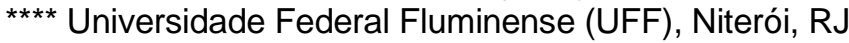

\begin{abstract}
Resumo: No Brasil, a Análise do Impacto Regulatório (AIR) vem se consolidando nas últimas décadas nas agências reguladoras federais. A metodologia AIR tem como objetivo examinar o processo regulatório, medir os custos e os benefícios gerados, assim como outros efeitos de natureza social, política ou econômica que podem ser causados por uma regulação nova ou já existente. Ao analisar cada opção regulatória, o especialista trata variáveis de natureza qualitativa de difícil mensuração e de elevado grau de incerteza. O trabalho complementa a literatura existente, dado que poucos trabalhos no âmbito da AIR têm empregado metodologias de apoio à decisão que incorporem o conhecimento do especialista de forma independente do problema a ser tratado. Portanto, propõe-se uma abordagem exploratória usando um Sistema Especialista Fuzzy (SEF), o qual, incorporando o conhecimento tácito dos especialistas, contribui para enriquecer o processo de decisão na fase final de comparação das opções regulatórias, permitindo o acesso do tomador de decisão a um histórico de parte dos raciocínios de outros especialistas.
\end{abstract}

Palavras-chave: Análise de Impacto Regulatório. Lógica Fuzzy. Sistema Especialista Fuzzy. Opção regulatória.

\begin{abstract}
Regulatory Impact Analysis (RIA) has been consolidating in Brazilian regulatory agencies throughout the last decades. The RIA methodology aims to examine the regulatory process, measure the costs and benefits generated, as well as other effects of social, political or economic nature caused by a new or an existing regulation. By analysing each regulatory option, the expert or regulator faces a myriad of variables, usually of qualitative nature, that are difficult to measure and with a high degree of uncertainty. This research complements the existing literature, given the scarcity of decision support models in RIA that regardless of the problem treated - incorporate the tacit knowledge of the regulation expert. This paper proposes an exploratory approach using a Fuzzy Expert System, which therefore helps to enrich the decision process in the final stage of comparison of the regulatory options.
\end{abstract}

Keywords: Regulatory Impact Analysis. Fuzzy Logic. Fuzzy Expert System. Regulatory option. 


\section{INTRODUÇÃO}

O processo de tomada de decisão envolvendo a atividade regulatória requer a inferência de variados aspectos de caráter qualitativo e quantitativo, sendo usadas diversas técnicas na coleta, no processamento e na interpretação desses dados. Um método crescentemente empregado nesse âmbito é o da Análise de Impacto Regulatório (AIR). Algumas agências reguladoras no Brasil vêm aderindo a AIR (ANEEL, 2014; ANTT, 2013; ANVISA, 2008), cuja maior vantagem, segundo a Organização para a Cooperação e Desenvolvimento Econômico (1997), é ser uma ferramenta com maior compatibilidade com a avaliação de aspectos econômicos e sociais de interesse, os quais afetam o processo regulatório (EC, 2009; ELLIG; MCLAUGHLIN; MORRALL III, 2013; FRANCESCO; RADAELLI; TROEGER, 2012; JACOBS, 2006; OCDE, 1997; SALGADO; BORGES, 2010).

A metodologia AIR examina o processo regulatório, mede os custos e os benefícios gerados, entre outros efeitos que podem ser causados por uma regulação nova ou já existente. Portanto, a AIR representa uma coleção de diretrizes para análise, que se propõe como uma maneira de auxiliar os tomadores de decisão envolvidos em um processo regulatório.

O processo de AIR lida com um volume substancialmente elevado de informação. Interessados em propor modelos interpretativos capazes de processar muitas informações e critérios, diversos autores vêm propondo a aplicação de técnicas para melhorar a eficiência do processo de tomada de decisão no âmbito regulatório (RAGONA; MAZZOCCHI; ALLDRICK, 2012; RAUEN, 2011; SALGADO; BORGES, 2010), dessa forma complementando o processo proposto para a AIR com técnicas de apoio à decisão.

Devido à complexidade inerente à AIR, cujo escopo enfatiza a importância da integração de características subjetivas ao processo decisório, como os benefícios gerados por um impacto regulatório, abre-se caminho para novas metodologias de análise que incorporem o conhecimento dos especialistas envolvidos na identificação e na organização das variáveis que impactam um processo de regulação econômica (RAGONA; MAZZOCCHI; ALLDRICK, 2012). Logo, as experiências e o conhecimento 
tácito do especialista são elos inerentes ao processo de tomada de decisão, que podem afetar o processo da AIR.

A Lógica Fuzzy (Nebulosa) permite tratar um número elevado de variáveis qualitativas e quantitativas (ROSS, 2010). Ainda, a Lógica Fuzzy apresenta-se como uma forma de representar o conhecimento que possui determinado nível de subjetividade (SIMÕES; SHAW, 2007). Trata-se de uma característica típica dos elementos que integram um processo como o de AIR com suas variáveis de natureza econômica, social e política.

No âmbito da Lógica Fuzzy, um conceito de particular interesse para o presente trabalho é o de Sistema Especialista Fuzzy (SEF). SEFs utilizam a Lógica Fuzzy para reproduzirem raciocínios de especialistas em um sistema computacional, permitindo que o computador aja como eles na resolução de problemas (KRISHNAMOORTHY; RAJEEV, 1996).

Este trabalho tem como objetivo discutir as bases conceituais de um SEF para aplicação na AIR. Para tanto, são definidas as variáveis essenciais para o modelo de decisão em impacto regulatório, a fim de que se possa explicitar o conhecimento dos especialistas após já ter sido feita a análise de custo-benefício de cada opção regulatória pelos reguladores. O SEF proposto neste trabalho preenche duas lacunas importantes no processo de AIR: i) possibilita o armazenamento de parte do conhecimento tácito envolvido no processo de decisão da melhor opção; e ii) habilita a incorporação de conhecimento novo ao modelo de decisão, por meio de técnicas de aprendizado por exemplo (learning by example).

O trabalho está organizado em cinco seções, incluindo esta Introdução. A seção 2 apresenta uma revisão da literatura da Análise de Impacto Regulatório e das técnicas empregadas atualmente nessa área. A seção 3 apresenta a metodologia da pesquisa e discute as bases conceituais do modelo de decisão proposto. A seção 4 discute os resultados e características do modelo proposto. Por fim, a seção 5 apresenta as conclusões. 


\section{ETAPAS E TÉCNICAS DA ANÁLISE DE IMPACTO REGULATÓRIO}

A AIR é uma prática difundida em diversos países (CELEBI, 2014; ELLIG; MCLAUGHLIN; MORRALL III, 2013; FRANCESCO; RADAELLI; TROEGER, 2012; OCDE, 2012; OMB, 2010; RAGONA; MAZZOCCHI; ALLDRICK, 2012). O fruto desses trabalhos vem se assimilando aos processos de decisão no âmbito regulatório tanto em termos de efetividade de uso dos recursos quanto de uma avaliação dos critérios pertinentes ao processo de tomada de decisão (ALVES; PECI, 2011). Trata-se, portanto, de uma abordagem de ampla aplicação no contexto das agências reguladoras, que as auxilia na garantia de decisões que expressem o cumprimento de determinações legais, como a promoção do bem-estar dos consumidores e do nível adequado de concorrência (SALGADO; BORGES, 2010).

A OCDE desenvolveu um guia a partir do resultado da experiência de seus países-membro com o intuito de recomendar boas práticas para AIR (OCDE, 1997). No âmbito da AIR, o primeiro passo é identificar o problema. Trata-se de uma etapa importante: se a caracterização do problema for equivocada, toda a condução da AIR é prejudicada. Devem-se ressaltar os sintomas do problema, o seu contexto, sua escala e suas tendências para em seguida poder descrever seus impactos negativos e positivos, assim como suas causas.

O segundo passo é estabelecer os objetivos políticos, nomeadamente a resolução dos impactos do problema após determinada política entrar em vigor. Assim, nessa etapa, é desenvolvida a lista de impactos que o regulador considera relevante solucionar.

No terceiro passo, que consiste em definir as opções de solução do problema, deve ser considerado o conjunto de opções pertinentes ao decisor: fazer nada; melhorar uma regulação existente (se houver); ou usar intervenções alternativas indiretas.

O quarto passo, o da consulta pública, é essencial para o bom andamento da AIR. Nesse estágio, os stakeholders tomam ciência do projeto de AIR, podendo contribuir com novas informações de impactos ou soluções. Ao fim, o quinto passo, o relatório de Análise de Impacto Regulatório é escrito com todas as informações dos passos anteriores. 
Esse processo em cinco passos é uma etapa inicial que antecede a adoção de uma regulação. Após escolhida uma opção regulatória, a mesma também deve ser monitorada, como é visto no trabalho de Silva (2014).

As agências reguladoras utilizam nomenclaturas muito similares, cujo entendimento é importante para a elaboração de um modelo de AIR. Na base do processo de avaliação, tem-se a Agenda Regulatória, de onde se originam os chamados Temas Regulatórios. A partir dos Temas Regulatórios, são obtidas as propostas de Ações Regulatórias. Por sua vez, essas propostas almejam objetivos que podem ser alcançados por Opções Regulatórias.

Em uma Análise de Impacto Regulatório, são avaliadas opções sobre uma determinada proposta regulatória. No Brasil, a Agência Nacional de Vigilância Sanitária (ANVISA) e a Agência Nacional de Transportes Terrestres (ANTT) envolvem os exemplos, até o momento, mais bem documentados de esforços de implementação da AIR. Vale ressaltar que cada agência reguladora possui seus próprios métodos de avaliação e, muitas delas, ainda estão desenvolvendo e aperfeiçoando seus métodos (RAUEN, 2011).

Na experiência da ANVISA, nos casos em que os impactos de uma regulação sejam considerados relevantes aos atores críticos (Sistema Único de Saúde - SUS, sociedade, entre outros), são aplicadas as pré-consultas, por meio de questionários, com os órgãos do Ministério da Saúde e da vigilância sanitária dos estados e municípios, entre outros (ANVISA, 2008).

Os dados provenientes dos questionários são usados para calcular o Índice de Governança e Impacto Regulatório (I-Reg), que monitora determinado programa, sob a perspectiva de qualidade do processo de regulamentação, da governança da atuação regulatória e do nível de impacto de cada regulamento (ANVISA, 2008).

Os níveis de qualidade que uma regulação pode apresentar, sob o ponto de vista do I-Reg (ANVISA, 2008), são os seguintes: i) considera-se o resultado ruim ou péssimo quando a avaliação indicar a preponderância de ameaças; ii) considera-se o resultado insuficiente quando a avaliação indicar a preponderância de pontos fracos; iii) considerase o resultado satisfatório quando indicar a preponderância de oportunidades; iv) 
considera-se o resultado excelente quando a avaliação indicar a preponderância de pontos fortes.

A ANTT, em 2009, em virtude da elaboração de seu planejamento estratégico, escolheu a AIR como um de seus projetos estruturantes (ANTT, 2013). Um elemento principal é o Formulário de Análise Preliminar de Impacto Regulatório (FAPIR) ${ }^{1}$. Esse formulário orienta o tomador de decisão na estruturação dos principais fatores que influenciam suas opiniões quanto a um determinado tipo de regulação. Os principais impactos oriundos das regulações considerados pela ANTT são: contratual, ambiental, social, fiscal, normativo e econômico, que, por sua vez, podem afetar os quatro agentes envolvidos: a sociedade, o prestador de serviço, o usuário e a agência reguladora.

Cada impacto, segundo a ANTT (2013), faz parte de um conjunto de aspectos, tais como, custos, benefícios e riscos, sendo os agentes envolvidos aqueles afetados pelos impactos de uma determinada opção regulatória. Vale ressaltar que os demais agentes também podem sofrer ou usufruir dos mesmos impactos, e isso dependerá do tipo de proposta que se quer implementar.

\subsection{Principais Técnicas do Processo de AIR}

O processo de AIR faz uso de uma gama de variáveis qualitativas e quantitativas. As variáveis qualitativas, segundo Ragona, Mazzocchi e Rose (2012), normalmente referem-se aos benefícios, de natureza reconhecidamente incerta. Tal abordagem é diferente no caso dos custos, os quais normalmente são expressos de forma quantitativa.

Uma das técnicas mais utilizadas no âmbito da regulação econômica é a Análise de Custo-Benefício (ACB). Na ACB devem ser incluídos e estratificados todos os impactos significativos da regulação, abrangendo aspectos que podem ser expressos monetariamente e os que não podem. As tipologias de ACB, resumidas no Quadro 1, podem ser encontradas em Salgado e Borges (2010). Um estudo aprofundado de ACB aplicado na AIR encontra-se em uma publicação da Comissão Europeia (EC, 2013).

${ }^{1}$ O FAPIR é dividido em 15 partes, sendo que a parte 8 desse processo, Análise das Opções, representa o ponto focal do presente trabalho.

Revista Produção Online, Florianópolis, SC, v.15, n. 3, p. 859-885, jul./set. 2015. 
Quadro 1 - Tipologias de ACB

\begin{tabular}{|l|l|}
\hline \multicolumn{1}{|c|}{ Tipologia } & \multicolumn{1}{c|}{ Descrição } \\
\hline Análises individuais & $\begin{array}{l}\text { Variam muito em rigor e se baseiam em pressupostos diferentes, } \\
\text { incluindo cenários de referência, métodos e dados. Alguns benefícios e } \\
\text { custos são de difícil avaliação, ou são de impossível quantificação ou } \\
\text { monetização. Para fins políticos, tais benefícios e custos que não são } \\
\text { quantificáveis podem ser importantes. Os mesmos, em algumas normas, } \\
\text { têm elementos significativos que servem como fatores-chave na decisão } \\
\text { de uma agência de promulgar uma regra específica. }\end{array}$ \\
\hline Análises prospectivas & $\begin{array}{l}\text { Podem vir a superestimar ou subestimar os benefícios e os custos; } \\
\text { análise retrospectiva, complementarmente, pode ser importante como } \\
\text { um mecanismo corretivo a fim de promover uma direção para a } \\
\text { regulamentação. }\end{array}$ \\
\hline
\end{tabular}

Fonte: Adaptado de Salgado e Borges (2010)

Uma abordagem encontrada em avaliações do tipo da AIR é a Análise Multicritério. Recomendada pela OCDE (2008), a Análise Multicritério considera os diversos critérios de natureza multidimensional envolvidos no processo de decisão envolvendo opções regulatórias distintas, tais como, políticas alimentares, ambientais, entre outras (RAGONA; MAZZOCCHI; ALLDRICK, 2012). Uma adaptação da Análise Multicritério para tratar as variáveis qualitativas do processo regulatório foi elaborada por Ragona, Mazzocchi e Alldrick (2012) e aplicada em um conjunto de políticas regulatórias na União Europeia.

O tratamento de variáveis qualitativas encontra-se entre os maiores desafios nos modelos baseados em Análise Multicritério (SAATY, 2005). Trata-se de um desafio de modelagem e de interpretação de resultados, devido às dificuldades de mensuração e comparabilidade dessas variáveis.

\subsection{A utilização da Teoria Fuzzy na AIR}

A Lógica Fuzzy surgiu a partir de esforços de entender como lidar com a imprecisão em processos decisórios. A imprecisão pode ser usada para descrever certos tipos de incertezas associadas a informações linguísticas ou intuitivas (ROSS, 2010; MELLO; SANTELLO, 2012). Dos estudos da imprecisão na tomada de decisão, surge o conceito de pertinência em conjuntos fuzzy. 
A ideia proposta por Zadeh (1965) sugere a pertinência de um elemento a um conjunto como a chave para a tomada de decisão sob incerteza. Um conjunto fuzzy estende a noção binária de um conjunto clássico. Essa propriedade é expressa pela função de pertinência $\mu(x)$, cujos valores estão dentro do intervalo $[0,1]$. Tal abordagem introduziu a possibilidade de um elemento ser membro parcial de um conjunto, indicado por valores intermediários no intervalo $[0,1]$.

Outro conceito importante no âmbito da Lógica Fuzzy é o de variável linguística. Variáveis linguísticas são aquelas cuja definição é dada por um conjunto de termos, nomes ou rótulos que podem ser associados a conjuntos fuzzy específicos (ZADEH, 1965).

Em um trabalho visando à solução de um caso específico de AIR, Ragona, Mazzocchi e Alldrick (2012) aplicam a Análise Multicritério Fuzzy, a qual possibilita um acompanhamento da avaliação dos impactos regulatórios, de maneira discreta e qualitativa. A ferramenta utilizada na Análise Multicritério Fuzzy ordena suas etapas da seguinte forma: análise qualitativa; filtro de viabilidade e avaliação quantitativa; e comparação das opções políticas.

A Análise Qualitativa é o primeiro passo da Análise Multicritério Fuzzy, usada para pontuar cada impacto das opções políticas baseando-se numa escala qualitativa. Essa pontuação é obtida por intermédio da combinação de quatro componentes de impacto: i) direção do impacto político (se positivo, neutro ou negativo); ii) severidade (medido pela intensidade do efeito aos agentes afetados pela política); iii) escala (proporção da população afetada pela política); e iv) likelihood (probabilidade do impacto se materializar).

Em seguida, a etapa do filtro de viabilidade determina a oportunidade de avaliar quantitativamente alguns dos impactos do método, considerando: i) a disponibilidade de informação primária ou secundária para comparação qualitativa; ii) os custos necessários para coleta quantitativa para efetuar a avaliação quantitativa em comparação com as pesquisas disponíveis; e iii) a presença ou ausência de alguma restrição de tempo para realizar a avaliação quantitativa.

$\mathrm{Na}$ última etapa, é feita a comparação entre as opções. Para tanto, os impactos qualitativos das opções são transformados em conjuntos gaussianos nebulosos. Já para 
os impactos quantitativos são atribuídas distribuições gaussianas padrão normalizadas. Após isso, as distâncias entre todos os pares de conjuntos nebulosos são computadas. A distância para cada par de opções políticas é calculada para cada impacto. Finalmente, dois indicadores para cada uma das opções políticas são computados. Eles podem ser interpretados como graus de pertinência para as afirmações: "esta opção é melhor" e "esta opção é pior". Segundo Ragona, Mazzocchi e Rose (2012), a metodologia fuzzy desenvolve um necessário indicador de credibilidade que resulta da combinação de i) uma escala de dominância de uma opção política versus outra (método de pareamento) para cada impacto individual; e ii) uma escala de incerteza em uma medição linguística. A avaliação do impacto individual e as comparações por pareamento são então agregadas para produzir um ranking de opções políticas.

\section{METODOLOGIA}

Esta seção apresenta os principais aspectos metodológicos da pesquisa e suas atribuições (subseção 3.1), também os detalhes da proposta do trabalho desenvolvido neste artigo (subseção 3.2).

\subsection{Aspectos da Pesquisa}

A pesquisa desenvolvida neste trabalho possui interesse prático, ou seja, objetiva que seus resultados sejam utilizados na prática, solucionando problemas observados nos contextos discutidos nas seções anteriores. Essa característica fornece a esta pesquisa um caráter de pesquisa aplicada (TURRIONI; MELLO, 2012).

Sob o ponto de vista do objetivo da pesquisa, pode-se classificá-la como exploratória, pois se busca o melhor entendimento do problema, para então gerar hipóteses e soluções (TURRIONI; MELLO, 2012).

Pode-se ainda classificar esta pesquisa quanto à maneira de tratar o problema. Nesse caso, ela pode ser considerada qualitativa, pois trata de aspectos subjetivos de um determinado problema (e.g. como uma pontuação de custo-benefício irá influenciar em uma avaliação geral de uma opção regulatória?).

Revista Produção Online, Florianópolis, SC, v.15, n. 3, p. 859-885, jul./set. 2015. 
A metodologia utilizada é denominada estudo de caso, pois se estuda um processo específico, que é a escolha entre opções regulatórias (TURRIONI; MELLO, 2012).

As técnicas de coleta de dados utilizadas neste trabalho se baseiam na pesquisa documental, principalmente por meio de notas técnicas e documentos específicos oriundos da execução dos fluxos informacionais dos processos de análise de impacto regulatório, da ANTT e da ANVISA, duas agências reguladoras de âmbito federal, os quais são adaptados para uso no processo de construção do modelo proposto no trabalho. O processo de trabalho foi amparado por entrevistas realizadas em junho de 2013 na sede da ANTT em Brasília com dois especialistas em regulação da área de Gestão de Atos Normativos da unidade organizacional responsável pela manutenção do Marco Regulatório da Agência e onde se localizam os conhecimentos mais relevantes relacionados aos processos de análise de impacto regulatório. $O$ tipo de entrevista foi a semiestruturada, com perguntas abertas e fechadas visando registrar e compreender como funciona a etapa de análise do impacto regulatório e posteriormente o processo de decisão no que tange à aprovação dos atos normativos da agência. A AIR foi um dos temas abordados no âmbito da gestão da informação e do conhecimento na agência, como parte de um projeto estruturante na agência denominado Modelo de Gestão da Informação e do Conhecimento (MGIC) (BASTOS et al., 2011; BASTOS et al., 2013), desenvolvido pelo grupo de pesquisa dos autores do presente trabalho. Como resultado da coleta de dados e de um processo de Engenharia de Software, foram produzidos casos de uso e um diagrama de informações relativo à AIR, os quais geraram a base de informações e de conhecimento do processo da AIR em uma agência reguladora.

Assim, é possível dividir a pesquisa em três etapas: i) inicia-se pela pesquisa exploratória, desenvolvendo as bases para criação de um modelo; ii) etapa da criação do SEF em si; e iii) validação do modelo. Ao longo da construção do Sistema Especialista Fuzzy, a fim de simular e calcular os resultados, utilizou-se a ferramenta Fuzzy Inference System (FIS) do Matlab. Os resultados gerados devem ser validados de modo a se garantir melhor qualidade dos resultados obtidos. Contudo, não foi possível contar com o apoio dos especialistas nessa etapa de validação. Visando contornar esse aspecto, tendo 
em vista a validade do presente trabalho como esforço de pesquisa empírica, optou-se pela estratégia de realização de dois testes, conforme exposto na seção 4.

\subsection{Proposta de um Modelo Fuzzy na AIR}

A elaboração do trabalho considera a investigação dos principais elementos inerentes ao processo regulatório das agências brasileiras (ANVISA, 2008; ANTT, 2013), assim como outras fontes que apresentam propostas de soluções para problemas envolvendo a AIR (PECI, 2009; RAGONA; MAZZOCCHI; ROSE, 2012; RAUEN, 2011).

A arquitetura básica de um SEF é composta de um Fuzzificador, módulo que mapeia as variáveis de entrada para valores fuzzy; outro módulo é a Base de Conhecimento, onde são armazenadas a base de parâmetros do modelo e definidas as T-normas, T-conormas e funções de pertinências. Há também a Base de Regras, onde o raciocínio lógico do processo de inferência é estruturado. O último módulo é o Defuzzyficador, cujo papel é traduzir a métrica dos resultados fuzzy obtidos para que seja possível atingir uma interface com o usuário.

Os tipos de SEF mais conhecidos na literatura são o tipo Mamdami (MAMDANI, 1977) e o Takagi-Sugeno (TAKAGl; SUGENO, 1985). O primeiro modelo tem como característica principal modelar processos levando em consideração entradas e saídas no formato de variáveis linguísticas, enquanto o segundo tem como principal característica a entrada como variável linguística e a saída uma função da combinação linear das consequências das regras ativadas.

Sistemas Especialistas Fuzzy são muito úteis em dois contextos gerais (ROSS, 2010): i) os que envolvem sistemas altamente complexos, cujos comportamentos não são bem compreendidos; e ii) em situações em que uma solução aproximada, porém rápida, é justificada.

Nas agências reguladoras, a obtenção da melhor opção regulatória é feita em

vários passos, como visto na seção 2. Identificados os impactos de cada opção, fazem-se comparações entre os vários impactos envolvendo cada opção, como pode ser visto nas linhas contínuas da Figura 1. 
Figura 1 - Diagrama esquemático segundo cada opção regulatória Opçāo 1

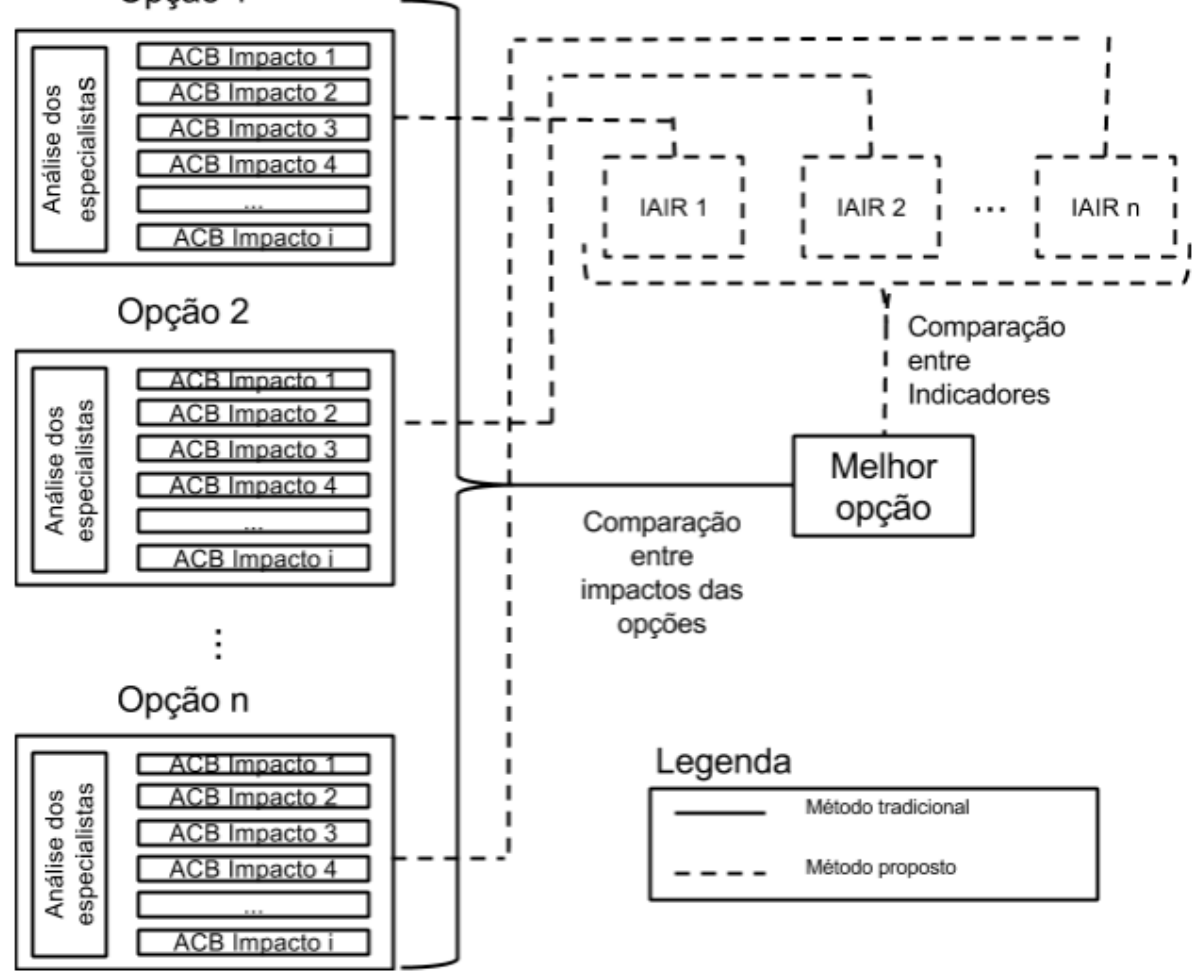

Fonte: Dados da pesquisa

A Figura 1 apresenta, em linhas tracejadas, outro modo de se chegar à melhor opção. O sistema recebe como entrada a ACB para cada impacto $i$, sendo $i$ um conjunto de notas elaboradas pelo especialista. A saída do sistema é o Índice de Análise de Impacto Regulatório (IAIR) para cada opção. Esse procedimento é repetido para todas as opções regulatórias, sendo $n$ o número de opções regulatórias consideradas. No final, a opção com o maior índice de AIR será a melhor. Observa-se que o processo facilita a escolha da melhor opção, visto que a comparação entre índices é mais parcimoniosa que o método tradicional. Ademais, permite que o especialista tenha um raciocínio com fatores qualitativos, por exemplo: uma entrada com $A C B$ baixo, ou $A C B$ entre baixo e médio, ou ACB com maior pertinência ao médio e um pouco de ACB baixo.

$\mathrm{Na}$ abordagem aqui proposta, a avaliação das opções é feita pelos especialistas. O SEF os auxilia, gerando notas que sintetizam a informação obtida dos impactos avaliados para determinada opção regulatória, proporcionando ainda que o especialista interprete o resultado do sistema através da base de regras utilizada pelo modelo. 
Para o desenvolvimento da proposta, são consideradas duas decisões: escolha das variáveis ACB e IAIR e avaliação do número de regras do modelo.

Para um SEF existem várias possibilidades de configurações de variáveis linguísticas para as mais variadas situações. Tal tipologia de variáveis vem definida por elementos chamados termos. Cada termo é definido por conjuntos fuzzy que, por sua vez, vêm definidos pelo formato de sua função de pertinência. Segundo Simões e Shaw (2007), os formatos mais frequentes de funções de pertinência são triângulos e trapezoides, pois são gerados com maior facilidade, embora outros formatos possam ser utilizados.

O modelo proposto trabalha com os resultados das ACBs para cada um dos seis impactos, os quais foram escolhidos de acordo com os seis impactos da ANTT (2013). Devido à sua natureza mista, visando trabalhar tanto com variáveis quantitativas quanto qualitativas, optou-se por considerar a representação por trapézios, conforme a Figura 2. Foram definidos os conjuntos fuzzy genéricos para todas as variáveis de entrada, usando três termos linguísticos: baixo, médio e alto.

Figura 2 - Variável de entrada que representa a ACB de cada impacto regulatório

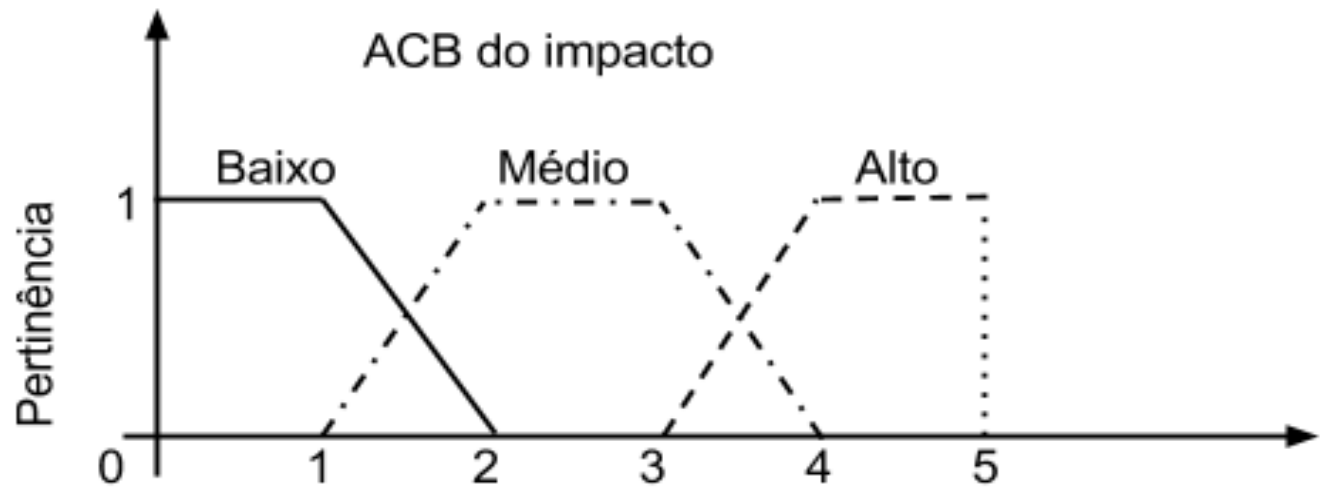

Fonte: Dados da pesquisa

A variável de saída IAIR foi modelada com base no I-Reg (ANVISA, 2008), o qual contém os quatro termos de avaliação (Ruim, Insuficiente, Satisfatório e Excelente), conforme Figura 3. 
Figura 3 - Variável de saída que representa um índice de AIR

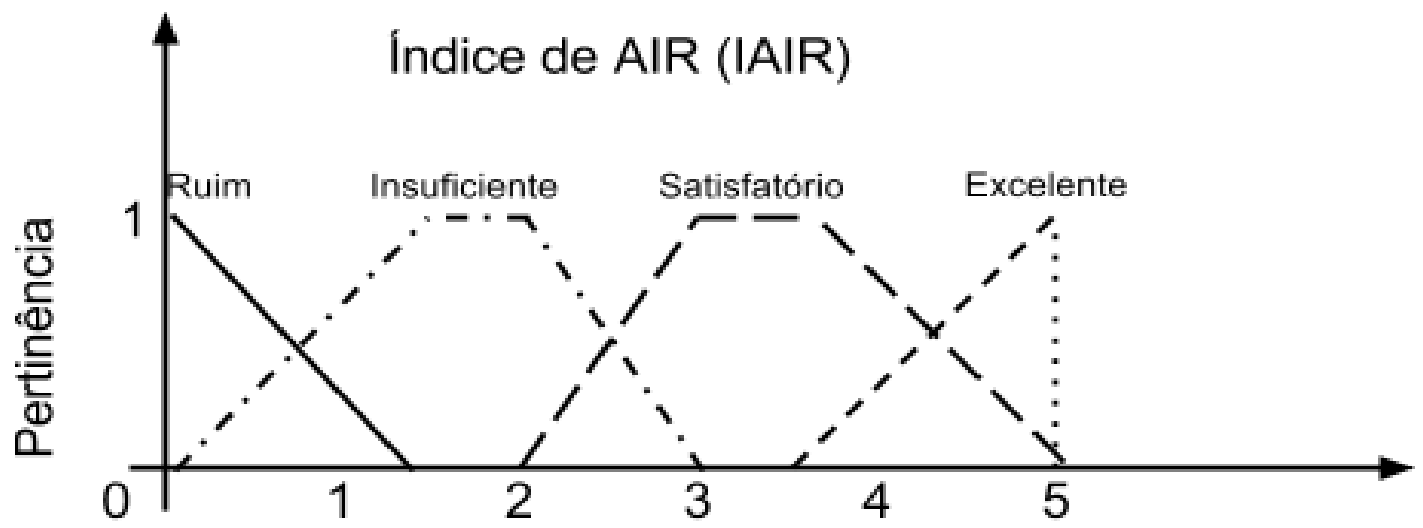

Fonte: Dados da pesquisa

A estratégia escolhida consistiu na avaliação de cada opção regulatória, levando em consideração os índices gerados pelo SEF. Como descrito na Figura 1, o modelo se caracteriza pelas variáveis de entrada - ACB dos respectivos impactos - e a variável de saída - o IAIR.

A escolha das Variáveis de Entrada (VEs) terá impacto na quantidade de regras do modelo, dado que um número de regras alto compromete o desempenho do sistema e a interpretação das regras, tornando difícil a compreensão de como ocorre a inferência (COPETTI et al., 2013).

Ainda, o número de regras deve ser pormenorizadamente avaliado na fase de construção do modelo de AIR. Um número de regras excessivo pode tornar o modelo mais complexo, afetando a sua interoperabilidade.

A Tabela 1 ilustra a quantidade de regras possíveis em um SEF, de acordo com o número de variáveis de entrada, separadas por subsistemas. O cálculo da quantidade de regras e do total de VEs de cada configuração da Tabela 1 é feito através da Equação 1 e da Equação 2, a seguir:

\footnotetext{
$\mathrm{N}^{\mathrm{o}}$ de regras $=\left(\mathrm{N}^{\mathrm{o}}\right.$ de subsistemas $) \times(\text { Termos })^{\mathrm{VE}}$

Equação 1

Total de VEs $=($ VEs por subsistema $) \times\left(N^{0} \text { de subsistemas }\right)^{\mathrm{VE}}$

Equação 2
} 
Tabela 1 - Número de regras em relação ao número de VEs e quantidade de subsistemas

\begin{tabular}{ccccc}
\hline VEs por subsistemas & Termos (Rótulos) & $\begin{array}{c}\text { Número } \\
\text { subsistemas }\end{array}$ & Total de VEs & $\begin{array}{c}\text { Número de } \\
\text { regras }\end{array}$ \\
\hline 2 & 3 & 1 & 2 & 9 \\
\hline 2 & 3 & 2 & 4 & 18 \\
\hline 2 & 3 & 3 & 6 & 27 \\
\hline 3 & 3 & 4 & 12 & 108 \\
\hline 3 & 3 & 8 & 24 & 216 \\
\hline 5 & 3 & 9 & 45 & 8787 \\
\hline 6 & 3 & 12 & 72 & 8748 \\
\hline
\end{tabular}

Fonte: Dados da pesquisa

A Tabela 2 apresenta a relação entre variáveis de entrada e termos do modelo com o número de regras possíveis. O cálculo da quantidade de regras para cada configuração de modelo na Tabela 2 é feito a partir da Equação 3, a seguir:

$$
\mathrm{N}^{\mathrm{o}} \text { de regras }=(\text { Termos })^{\mathrm{VE}} \quad \text { Equação } 3
$$

Tabela 2 - Relação entre número de VEs e termos com a quantidade de regras

\begin{tabular}{ccc}
\hline VEs & Termos (Rótulos) & Número de regras \\
\hline 2 & 3 & 9 \\
4 & 3 & 81 \\
6 & 3 & 729 \\
\hline 12 & 3 & 531441 \\
\hline 48 & 3 & $7,98 E+22$ \\
\hline 74 & 3 & $2,03 E+35$ \\
\hline
\end{tabular}

Fonte: Dados da pesquisa

Um impacto regulatório advém de vários aspectos. A ANTT (2013) estabelece um mínimo de seis desses aspectos (social, ambiental, econômico, normativo, fiscal e contratual) que podem afetar os quatro agentes envolvidos (a sociedade, o prestador de serviço, o usuário e a agência reguladora).

Se fossem levados em consideração todos os benefícios, custos e riscos dos impactos de todos os agentes, ter-se-ia um total de 72 variáveis no modelo: 4 agentes $\times 3$ consequências $\times 6$ impactos.

Após analisar essa configuração, optou-se por um modelo que utiliza os custos e benefícios no que tange aos impactos mínimos a serem considerados na AIR. A partir disso, notou-se que o custo e o benefício de um determinado impacto a um determinado agente podem ser mais facilmente interpretados se tratados em duplas relacionadas. Por 
exemplo, em uma regra que tenha o benefício do impacto econômico para a prestadora de serviços, deve-se ter, impreterivelmente, o custo do mesmo impacto.

Dessa forma, o especialista leva em consideração dois aspectos: benefício e custo do impacto econômico, para então gerar uma nota de custo-benefício referente, única e exclusivamente, ao mesmo impacto. Essa premissa reduz, significantemente, o número de regras possíveis, visto que o custo do impacto ambiental não afeta na nota do custobenefício do impacto econômico. Assim, cada variável de entrada se torna um par custobenefício e as 72 variáveis de entrada se tornam 6, utilizando a ACB dos impactos de todos os agentes. Como em toda simplificação analítica, essa última configuração deixa de levar em consideração certos aspectos, como o risco do impacto, o qual pode ser levado em consideração em avaliações subsequentes.

As regras foram feitas por meio da combinação de todos os termos das variáveis de entrada, totalizando 729 regras. Ou seja, três termos e seis variáveis linguísticas, produzindo $3^{6}=729$ regras.

\subsubsection{Fases da proposta de um sistema fuzzy para AIR}

Esta subseção estabelece a visão do processo de tomada de decisão em três fases.

Primeira fase do processo - o especialista efetua a avaliação dos impactos de uma opção regulatória através do método ACB. A Tabela 3 mostra um exemplo de resultado fornecido pelo especialista na primeira fase: uma série de notas correspondentes às diversas intensidades de impactos que podem ser ocasionadas por uma determinada opção. Essas pontuações são a entrada do modelo.

Tabela 3 - ACB e o resultado da análise a ser feita por um especialista

\begin{tabular}{cccccc}
\hline Econômico & Social & Contratual & Normativo & Ambiental & Fiscal \\
\hline 2,5 & 3 & 1,75 & 5 & 4 & 2 \\
\hline Fonte: Dados da pesquisa & & & &
\end{tabular}

Segunda fase do processo - o especialista desenvolve o raciocínio de avaliação dos resultados obtidos na primeira fase para obtenção da base de regras do SEF. Para isso, propõe-se uma Tabela de Critérios, conforme a Figura 4. O especialista deve Revista Produção Online, Florianópolis, SC, v.15, n. 3, p. 859-885, jul./set. 2015. 
especificar quais os impactos que apresentam pouca relevância e, em seguida, colocar os intervalos de quantidades que relacionam o resultado da ACB com o resultado da AIR. A Figura 4 mostra uma Tabela de Critérios já preenchida, mas que pode ser ajustada pelo especialista. Essa etapa gera automaticamente as 729 regras, originadas das combinações dos antecedentes (as ACBs). Considerando o quadro da Figura 4, para uma opção regulatória obter um Resultado de AIR Excelente, são necessários três ou mais impactos com ACB altos, entre aqueles considerados relevantes.

Figura 4 - Tabela de Critérios e a geração de regras

\begin{tabular}{|c|c|c|c|c|c|c|c|c|c|c|}
\hline \multicolumn{11}{|c|}{ Impactos de pouca relevância } \\
\hline Econômico & Social & \multicolumn{2}{|c|}{ Contratual } & \multicolumn{2}{|c|}{ Normativo } & Ambiental & Fiscal & \multicolumn{2}{|c|}{ Bloco 1} & \\
\hline & \multicolumn{9}{|c|}{ Resultado da opçäo regulatória } & \\
\hline \multirow{2}{*}{ Impactos } & \multicolumn{2}{|c|}{ Ruim } & \multicolumn{3}{|c|}{ Insuficiente } & \multicolumn{2}{|c|}{ Suficiente } & \multicolumn{2}{|c|}{ Excelente } & \multirow{3}{*}{ Bloco 2} \\
\hline & Operador & Valor & & erador & Valo & Operador & Valor & Operador & Valor & \\
\hline \multirow{2}{*}{ Baixo } & $>=$ & 4 & & $x=$ & 1 & & & & & \\
\hline & & & & $\Leftrightarrow$ & 3 & & & & & \\
\hline \multirow{2}{*}{ Médio } & & & & & & $>=$ & 2 & & & \\
\hline & & & & & & & & & & \\
\hline \multirow{2}{*}{ Alto } & & & & & & & & $x=$ & 3 & \\
\hline & & & & & & & & & & \\
\hline
\end{tabular}

Fonte: Dados da pesquisa

Observa-se no Bloco 1 da Figura 4 o mecanismo que possibilita a sensibilidade do modelo para desconsiderar determinados impactos especificados pelo especialista. A estratégia é utilizada quando um impacto específico $i$ é considerado de pouca relevância pelo especialista, marcando-se um ' $X$ ' no campo referente ao impacto $i$. O sistema aqui proposto faz um rastreamento das regras com antecedentes de valores altos e médios da ACB desse impacto $i$ que terão consequentes baseados apenas nos demais impactos; ou seja, as cinco ACBs restantes vão determinar o consequente da regra, seguindo a relação de impactos definido no Bloco 2 - Tabela de Critérios da Figura 4.

Terceira fase do processo - nesta fase, o mecanismo de inferência produz o resultado da opção regulatória. Para obter o resultado, a base de regras gerada é incluída em um arquivo FIS do Matlab, com a configuração do modelo, conforme a Figura 5. 
Figura 5 - Visão geral do modelo e suas etapas

Primeira fase

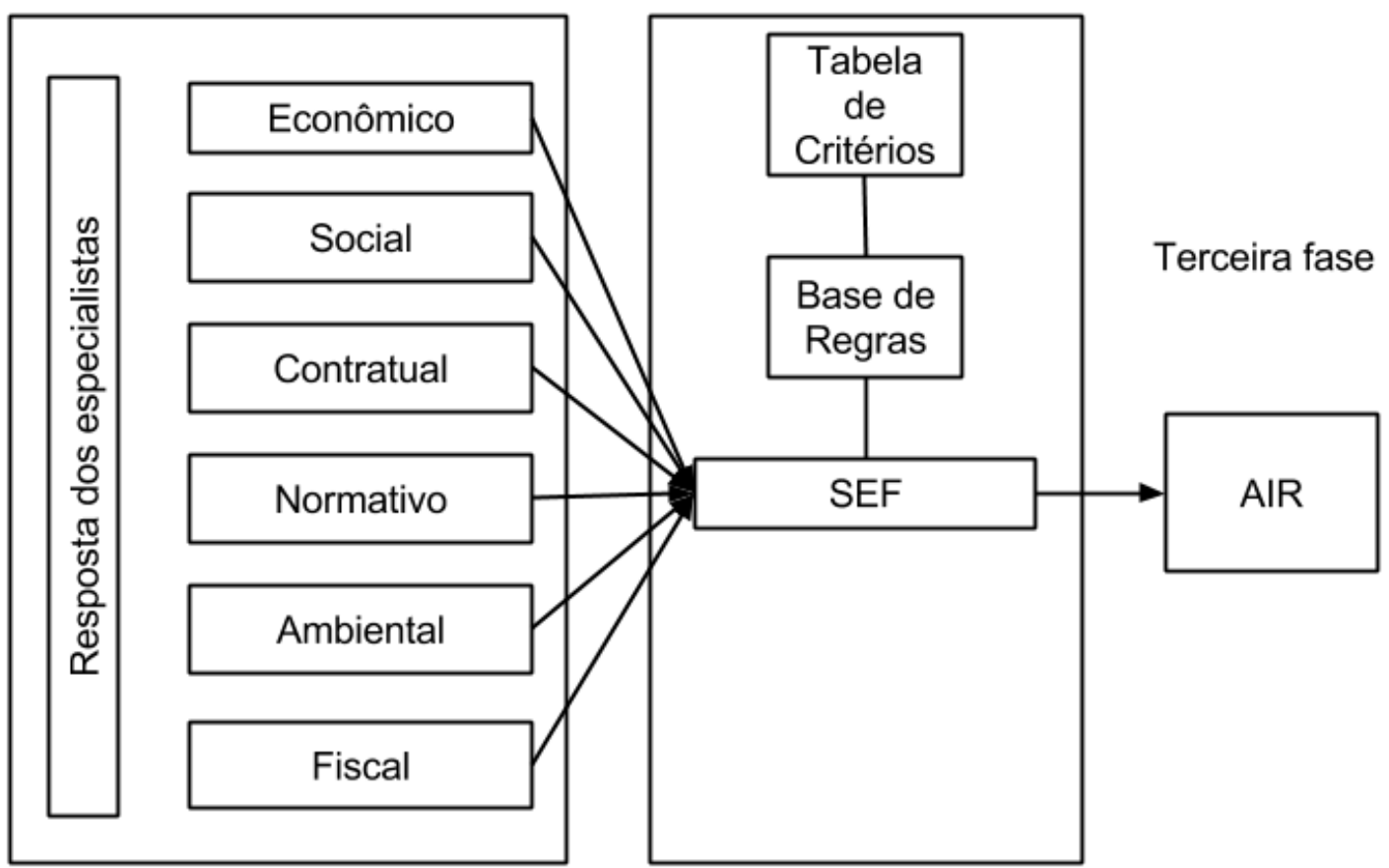

Fonte: Dados da pesquisa

A configuração do SEF foi aperfeiçoada durante o desenvolvimento do trabalho. Como a saída do modelo é linguística, definiu-se o SEF de arquitetura Mamdani. ${ }^{2}$

\section{TESTES E AVALIAÇÃO DA PROPOSTA}

Para avaliar a sensibilidade do modelo e validá-lo, foram elaborados dois testes com dados simulados. Um caso similar ao exposto nesta seção pode ser observado no relatório da ANVISA (2010), cuja proposta de regulamento técnico para o funcionamento dos bancos de células e tecidos germinativos é avaliada segundo os critérios determinados pela própria agência, conforme elaborado na seção 3.

Teste (A): avaliação entre seis opções de regulação em que cada uma possui uma nota de custo-benefício para cada um dos seis impactos.

2 A configuração final da base de parâmetros do modelo foi ajustada de maneira empírica: o operador 'implicação' escolhido foi o 'mínimo'; na 'agregação', o operador 'soma' foi utilizado; o 'centroide' foi o método de 'defuzzificação' empregado.

Revista Produção Online, Florianópolis, SC, v.15, n. 3, p. 859-885, jul./set. 2015. 
O especialista elabora o raciocínio de avaliação das relações através do Bloco 2 da Tabela de Critérios (Figura 4), permitindo que o processo posterior gere a base de regras do modelo.

Definiram-se todos os impactos como relevantes. Portanto, o Bloco 1 permaneceu com todos os campos vazios para a criação da base de regras, conforme a Tabela 4.

Tabela 4 - Bloco 1 - impactos de pouca relevância do Teste A

\begin{tabular}{|c|c|c|c|c|c|}
\hline Econômico & Social & Contratual & Normativo & Ambiental & Ficcal \\
\hline
\end{tabular}

Fonte: Dados da pesquisa

Após a geração da base de regras, o especialista informa ao Sistema Especialista Fuzzy as notas de cada impacto da Opção 1 até a Opção 6, conforme Tabela 5.

Tabela 5 - Notas da ACB da Opção 1 até a Opção 6 do Teste A

\begin{tabular}{|c|c|c|c|c|c|c|c|c|}
\hline $\begin{array}{l}\text { Opção } \\
\text { regulatória }\end{array}$ & Econômico & Social & Contratual & Normativo & Ambiental & Fiscal & IAIR & Faixa \\
\hline Opção 1 & 0 & 0 & 0 & 0 & 0 & 0 & 0,4833 & Ruim \\
\hline Opção 2 & 0,3 & 4 & 1,5 & 3,5 & 0,5 & 3 & 2,5626 & Satisfatório \\
\hline Opção 3 & 4,3 & 4 & 1,5 & 3,5 & 0,5 & 3 & 3,59 & Satisfatório \\
\hline Opção 4 & 0,5 & 4 & 1,5 & 0,5 & 0,5 & 3 & 2,3615 & Insuficiente \\
\hline Opção 5 & 4,3 & 4 & 1,5 & 0,5 & 0,5 & 3 & 2,5626 & Satisfatório \\
\hline Opção 6 & 5 & 5 & 5 & 5 & 5 & 5 & 4,5167 & Excelente \\
\hline $\begin{array}{l}\text { Pouca } \\
\text { relevância }\end{array}$ & Não & Não & Não & Não & Não & Não & & \\
\hline
\end{tabular}

Fonte: Dados da pesquisa

Os resultados produzidos são mostrados nas duas últimas colunas da Tabela 5. Percebe-se que há uma distância significativa no IAIR das opções 2 e 3 (uma distância de 1,0274 pontos), que acontece devido à expressiva diferença no ACB do Impacto Econômico.

Teste (B): Aqui o procedimento é diferenciado pelo fato de o especialista, no momento que antecede a geração da base de regras, desconsiderar um dos impactos, implicando uma mudança na base de regras. A Tabela 6 apresenta o campo do Bloco 1 Revista Produção Online, Florianópolis, SC, v.15, n. 3, p. 859-885, jul./set. 2015. 
com o impacto econômico preenchido com um ' $X$ ', orientando o sistema a desconsiderar valores altos e médios desse impacto na geração dos consequentes das regras do modelo.

Tabela 6 - Bloco 1 - impactos de pouca relevância para o Teste B

\begin{tabular}{|c|c|c|c|c|c|}
\hline Econômico & Social & Contratual & Normativo & Ambiental & Fiscal \\
\hline $\bar{X}$ & & & & & \\
\hline
\end{tabular}

As notas de cada impacto da Opção 1 até a Opção 6 seguem na Tabela 7.

Tabela 7 - Notas da ACB da Opção 1 até a Opção 6 do Teste B

\begin{tabular}{|c|c|c|c|c|c|c|c|c|}
\hline $\begin{array}{l}\text { Opção } \\
\text { regulatória }\end{array}$ & Econômico & Social & Contratual & rmativo & Ambiental & Fiscal & IAIR & Faixa \\
\hline Opção 1 & 0 & 0 & 0 & 0 & 0 & 0 & 0,4833 & Ruim \\
\hline Opção 2 & 0,3 & 4 & 1,5 & 3,5 & 0,5 & 3 & 2,5626 & Satisfatório \\
\hline Opção 3 & 4,3 & 4 & 1,5 & 3,5 & 0,5 & 3 & 2,5626 & Satisfatório \\
\hline Opção 4 & 0,5 & 4 & 1,5 & 0,5 & 0,5 & 3 & 2,3615 & Insuficiente \\
\hline Opção 5 & 4,3 & 4 & 1,5 & 0,5 & 0,5 & 3 & 2,5626 & Satisfatório \\
\hline Opção 6 & 5 & 5 & 5 & 5 & 5 & 5 & 4,5167 & Excelente \\
\hline $\begin{array}{l}\text { Pouca } \\
\text { relevância }\end{array}$ & Sim & Não & Não & Não & Não & Não & & \\
\hline
\end{tabular}

Fonte: Dados da pesquisa

Os resultados obtidos no Teste $\mathrm{B}$ se diferenciam do Teste $\mathrm{A}$ devido à orientação do especialista em estabelecer a pouca relevância a um critério em detrimento a outro. Consequentemente, não aparece diferença entre as opções 2 e 3.

Vale ressaltar que a diferença entre as opções 4 e 5 ocorre porque o Bloco 2, ao sofrer a interferência do Bloco 1, alterou diversas regras do modelo. A regra do Bloco 2 é clara, se houver 2 ou mais impactos relevantes com ACB médio, o índice de análise de impacto será considerado Suficiente. Por exemplo, no caso do primeiro teste, na opção 5 , é ativada a seguinte regra: se $A C B$ econômico for alto, $A C B$ social for alto, $A C B$ contratual for médio, ACB normativo for baixo, $A C B$ ambiental baixo e ACB fiscal médio, então o IAIR será Suficiente.

Revista Produção Online, Florianópolis, SC, v.15, n. 3, p. 859-885, jul./set. 2015. 
O Bloco 2 afeta a opção 4 do seguinte modo: se houver entre 1 e 3 impactos relevantes com ACB baixo, então o IAIR será Insuficiente. Nesse caso, a regra ativada pela opção 4 é: se $A C B$ econômico for baixo, $A C B$ social for alto, $A C B$ contratual for médio, ACB normativo for baixo, ACB ambiental baixo e ACB fiscal médio, então o IAIR será Insuficiente.

Para o segundo teste, a mesma opção 5 também é Suficiente porque a interferência do Bloco 1 do modelo, ao considerar o ACB econômico pouco relevante, modifica o critério para determinar o valor do índice. Por exemplo, a regra do Bloco 2 é clara: se houver 2 impactos relevantes com impacto médio, então o índice de análise de impacto regulatório será Suficiente, sendo que o impacto econômico, apesar de ser desconsiderado, não é considerado como ACB baixo, o que acarretaria na mesma resposta da opção 4. Ao contrário, o sistema coloca o impacto econômico como se os seus valores de ACB altos e médios tivessem efeito neutro na AIR.

Observou-se que, nos dois testes, o modelo está coerente com o raciocínio da Tabela de Critérios definida pelo especialista. Assim, essa configuração pode ser útil na tomada de decisão em AIRs futuras.

\subsection{Limitações e Considerações do Método Proposto}

A principal limitação do trabalho é não ter podido contar com a validação de especialistas em regulação. Isso ocorreu devido a motivações alheias à vontade dos autores. Apesar disso, abre-se espaço para que o modelo proposto possa ser utilizado pelos especialistas e aprimorado, permitindo comparações com outros modelos aplicados à AIR. Portanto, acredita-se que tais aspectos limitadores do trabalho não o invalidam como um esforço de pesquisa empírica, visto que se traz à luz uma metodologia que pode ser utilizada e posta à prova crítica em avaliações subsequentes.

O método proposto neste trabalho possui o intuito de complementar outros que envolvam avaliações com muitos critérios, tendo em vista as dificuldades que métodos de apoio à decisão multicritério podem apresentar ao lidar com quantidades substancialmente elevadas de critérios (BERZINS, 2009; SAATY, 1990). Visando contornar tais aspectos, Saaty (1990) sugere a combinação da mensuração absoluta com 
a mensuração relativa do Analytical Hierarchy Process (AHP) nos casos envolvendo muitos critérios, implicando o uso de clusters de critérios.

Um SEF, diferentemente da metodologia AHP, por exemplo, baseia-se em regras lógicas. Portanto, a limitação de modelagem em um SEF está na forma como se representa o raciocínio do especialista, o que pode ser avaliado com um teste de sensibilidade do modelo em vários cenários distintos.

Outra limitação do SEF é o fato de não haver uma maneira de medir a consistência da base de regras do sistema por meio de um índice, como acontece no método AHP (SAATY; TRAN, 2007). No entanto, é possível, com clareza considerável, conferir o raciocínio da resposta por meio da execução de exemplos que possam demonstrar a sensibilidade do SEF a diversos cenários. Por outro lado, Saaty e Tran (2007) chamam a atenção para que a utilização de metodologias híbridas, como Fuzzy AHP, seja feita com discernimento.

A proposta do presente trabalho consistiu em adotar um SEF como técnica complementar ao processo de avaliação dos impactos da ACB na AIR. O SEF pode atuar tanto sozinho ou como uma abordagem complementar à AHP ou Fuzzy AHP, permitindo uma comparação dos resultados gerados por cada método em termos de esforço computacional e efetividade de resultados, tendo em vista a relação custo-benefício inerente ao processo de análise de problemas envolvendo uma miríade de variáveis, como é o caso da AIR. Ainda, em função de os resultados provenientes de uma AIR poderem se traduzir facilmente em elementos qualitativos de decisão (ANTT, 2013; ANVISA, 2008; OCDE, 2008), que, portanto, requerem menor precisão na orientação dos especialistas, um modelo SEF, conforme já delineado por Ross (2010), pode ser uma alternativa igualmente funcional e de implementação menos custosa.

Ainda, o SEF possibilita a utilização, em futuros processos de AIR, de parte do conhecimento tácito de especialistas empregado em avaliações anteriores, visto que a base de regras pode ser reutilizada. Por fim, tal abordagem abre caminho para modelos subsequentes incorporarem novas regras por meio de metodologias de aprendizado por exemplos, como sugerido por Wang e Mendel (1991) e Koshyiama (2014).

Ao agente regulador cabe avaliar os trade-offs entre tempo, precisão e custos das ferramentas de apoio à AIR. Dependendo da precisão necessária para os resultados a 
serem obtidos, há a possibilidade de se obter resultados similares com diferentes ferramentas. Portanto, uma metodologia construída sob um SEF pode ser recomendada nos casos em que a precisão no resultado não seja tão necessária e que suas alternativas sejam de implementação mais custosa e demandem maior esforço de modelagem e de cálculo computacional.

\section{CONCLUSÃO}

A AIR, apesar de ter ganho espaço na agenda regulatória de países em desenvolvimento, é ainda uma metodologia recente no âmbito nacional, sendo sua implementação o maior desafio enfrentado pelas agências reguladoras brasileiras.

O processo de tomada de decisão por parte do gestor público envolvendo a AIR é demasiadamente complexo e envolve variáveis que, em conjunto, delimitam o contexto e a amplitude do problema de impacto regulatório a ser avaliado. Algumas propostas existentes na literatura preconizam o uso de metodologias como a análise de custobenefício e a análise multicritério para a solução de problemas de AIR. Neste trabalho, de natureza exploratória, optou-se por avaliar como seria o processo e quais resultados se esperaria de uma análise custo-benefício em um problema de AIR envolvendo um Sistema Especialista Fuzzy.

Nesse âmbito, é possível que a avaliação entre as opções envolvidas no processo de tomada de decisão possa ser feita sem a construção de um modelo. No entanto, isso pode tornar todo o processo demasiadamente complexo e custoso, visto que o especialista ou gestor público necessita comparar vários impactos entre várias opções, o que geralmente se dá num horizonte curto de tempo. Portanto, constatou-se que o modelo pode facilitar a decisão de qual seja a melhor entre as opções disponíveis, ainda podendo diminuir a relevância de determinados impactos, criando assim o próprio raciocínio de avaliação que é utilizado para fazer a AIR. O SEF projetado pode ser reutilizado, significando a utilização das opiniões de raciocínios passados para auxiliar na tomada de decisões presentes, podendo também ser aprimorado por métodos de aprendizado por exemplo. Tendo em vista tais aspectos, este trabalho propôs um modelo

para efetuar a AIR em opções regulatórias em que o especialista pode adaptar 0 Revista Produção Online, Florianópolis, SC, v.15, n. 3, p. 859-885, jul./set. 2015. 
raciocínio de análise para o modo que the seja mais conveniente, dados os condicionantes políticos, econômicos e sociais normalmente envolvidos na regulação econômica. O modelo desenvolvido neste trabalho recebe as notas das ACBs dos impactos como entrada de dados e utiliza o raciocínio do especialista no formato da base de regras, com o auxílio da tabela de critérios desenvolvida no trabalho, para gerar uma nota de AIR para uma opção.

O índice de AIR que o modelo proposto fornece para uma determinada opção é um valor contido em um dado intervalo de números reais (nesse caso, o intervalo [0..5]). Para o especialista poder comparar várias opções, ele deve executar o modelo de AIR para cada opção e assim comparar as notas do índice. Parte do conhecimento do especialista nessa avaliação é armazenada na base de regras, que pode ser alterada sempre que for necessário, permitindo uma adaptabilidade que possibilita ajudar o especialista em AIR.

Embora a eficácia dos modelos de AIR ainda se encontre em fase de consolidação, sobretudo em função da dificuldade dos modelos existentes em interpretar a miríade de variáveis e questões envolvidas em um processo dependente de condicionantes políticos, econômicos e sociais, este trabalho objetivou contribuir com o debate trazendo uma perspectiva complementar com aplicação no âmbito da regulação.

Pelo caráter de estudo exploratório deste trabalho, a apresentação e discussão dos fatores críticos a serem considerados em um modelo de AIR podem permitir que novos estudos formulem hipóteses que mereçam investigações mais amplas sobre o processo de tomada de decisão no âmbito da AIR, assim como seus condicionantes na agenda regulatória brasileira. A participação dos especialistas é um elemento importante desse elo e deve ser encorajada pelas agências reguladoras.

\section{REFERÊNCIAS}

AGÊNCIA NACIONAL DE ENERGIA ELÉTRICA (ANEEL). Desenvolvido por ANEEL. Disponível em: <http://www.aneel.gov.br>. Acesso em: out. 2014.

AGÊNCIA NACIONAL DE TRANSPORTES TERRESTRES (ANTT). Desenvolvido por ANTT. Disponível em: <http://www.antt.gov.br>. Acesso em: ago. 2013. 
AGÊNCIA NACIONAL DE VIGILÂNCIA SANITÁRIA (ANVISA). Guia de boas práticas regulatórias. Brasília, DF: ANVISA, 2008.

ANVISA, 2010.

Relatório preliminar de análise de impacto regulatório AIR nível 2. Brasília, DF:

ALVES, F. N. R.; PECl, A. Análise de impacto regulatório: uma nova ferramenta para a melhoria da regulação na Anvisa. Rev. Saúde Pública, São Paulo, v. 45, n. 4, p. 802-805, ago. 2011. http://dx.doi.org/10.1590/S0034-89102011000400023

BASTOS, C. A. M.; BRUNO, A. C. M.; GARCIA, A.; REZENDE, L.; CALDAS, M. A. F.; SANCHEZ, M. L.; SILVA FILHO, S. J. M. Managing information and knowledge: a proposal methodology for building an integrated model based on information assets identification. In: Conference on Knowledge Discovery and Information Retrieval and the International Conference on Knowledge Management and Information Sharing, 2013, Portugal. Proceedings... Portugal: IC3K, 2013. p. 520-525. http://dx.doi.org/10.5220/0004627305200525

BASTOS C. A. M.; REZENDE, L. S.; CALDAS, M. F.; GARCIA, A. S.; MECENA FILHO, S.; SANCHEZ, M. D.; CASTRO JR, J. L. P.; BURMANN, C. Building up a model for management information and knowledge: the case-study for a Brazilian regulatory agency. In: International Workshop on Software Knowledge, 2., 2011, Paris. Proceedings... Paris: SKY, 2011. p. 3-11. http://dx.doi.org/ 10.5220/0003695200030011

BERZINS, L. J. Avaliação de desempenho pelo AHP, através do superdecisions: caso Inmetro. 2009. Dissertação (Mestrado) - Programa de Pós-Graduação em Administração, Faculdades Ibmec, Rio de Janeiro, 2009.

CELEBI, D. A framework for an impact analysis of ADR implementation in Turkey. Transportation Research Board, 2014.

COPETTI, A. et al. A decision-making mechanism for context inference in pervasive healthcare environments. Decision Support Systems, v. 55, p. 528-537, 2013.

http://dx.doi.org/10.1016/i.dss.2012.10.010

ELLIG, J.; MCLAUGHLIN, P. A.; MORRALL III, J. F. Continuity, change, and priorities: The quality and use of regulatory analysis across US administrations. Regulation \& Governance, v. 7, n. 2, p. 153-173, 2013. http://dx.doi.org/10.1111/j.1748-5991.2012.01149.x

EUROPEAN COMMISSION (EC). Impact assessment guidelines. SEC, v. 92, 2009.

. Assessing the costs and benefits of regulation (study). 2013. Disponível em:

<http://ec.europa.eu/smart-regulation/impact>. Acesso em: out. 2014.

FRANCESCO, F.; RADAELLI, C. M.; TROEGER, V. E. Implementing regulatory innovations in Europe: the case of impact assessment. Journal of European Public Policy, v. 19, n. 4, p. 491511, 2012. http://dx.doi.org/10.1080/13501763.2011.607342

JACOBS, S. Regulatory impact analysis in regulatory process, method, and co-operation - lessons for Canada from international trends. Working Paper Series 026, Canadá, 2006. 
KOSHYIAMA, A. S. GPFIS: um sistema fuzzy-genético genérico baseado em programação genética. Rio de Janeiro: PUC-Rio, 2014.

KRISHNAMOORTHY, C. S.; RAJEEV, S. Artificial intelligence and expert systems for engineers. Boca Raton, FL: CRC Press, Inc., 1996.

MAMDANI, E. H. Application of fuzzy logic to approximate reasoning using linguistic synthesis. Computers, IEEE Transactions on, v. 100, n. 12, p. 1182-1191, 1977.

http://dx.doi.org/10.1109/TC.1977.1674779

MELLO, R.; SANTELLO, R. O uso de lógica fuzzy para avaliação de imóveis e exemplos de aplicação. Revista Produção Online, Florianópolis, v. 12, n. 4, p. 904-927, 2012.

OFFICE OF MANAGEMENT AND BUDGET (OMB). Report to congress on the benefits and costs of federal regulations and unfunded mandates on state, local, and tribal entities. Washington, DC: OMB, 2010.

ORGANIZAÇÃO PARA A COOPERAÇÃO E DESENVOLVIMENTO ECONÔMICO (OCDE). Regulatory impact analysis: best practice in OECD countries. Paris: OCDE Publishing, 1997.

. Introductory handbook for undertaking regulatory impact analysis (RIA). Paris:

OCDE Publishing, 2008.

Measuring regulatory performance: evaluating the impact of regulation and regulatory policy. Paris: OCDE Publishing, 2012.

PECl, A. Avaliação do impacto regulatório, experiências internacionais e potencialidades de adoção em contextos nacionais: o caso brasileiro. In: Congreso Internacional del CLAD sobre la Reforma del Estado y de la Administración Pública, 14., 2009. Anais... Salvador: CLAD, 2009.

RAGONA, M.; MAZZOCCHI, M.; ALLDRICK, A. J. Multi-criteria analysis for the impact assessment of food safety policies: the case of EU regulation on dietary arsenic. AIEAA, Trento, jun. 2012.

RAGONA, M.; MAZZOCCHI, M.; ROSE, M. An application of fuzzy multi-criteria decision aid to the impact assessment of food safety policy: alternative EU regulations on dioxins. Journal of Agricultural Economics, v. 63, n. 3, p. 743-749, 2012.

RAUEN, C. V. Metodologias de análise de impacto regulatório e a análise custo-benefício. Brasília, DF: Secretaria de Acompanhamento Econômico, Ministério da Fazenda, 2011.

ROSS, T. J. Fuzzy logic with engineering applications. 3. ed. Reino Unido: John Wiley \& Sons, 2010.

SAATY, T. L. How to make a decision: the analytic hierarchy process. European Journal of Operational Research, v. 48, n. 1, p. 9-26, 1990. http://dx.doi.org/10.1016/0377-2217(90)90057-I

. The analytic hierarchy and analytic network processes for the measurement of intangible criteria and for decision-making. In: FIGUEIRA, J.; GRECO S.; EHRGOTT, M. Multiple criteria 
decision analysis: state of the art surveys. New York: Springer Sciense+Business Media, 2005. p. 345-408.

SAATY, T. L.; TRAN L. T. On the invalidity of fuzzifying numerical judgments in the analytic hierarchy process. Mathematical and Computer Modelling, v. 46, n. 7, p. 962-975, 2007. http://dx.doi.org/10.1016/j.mcm.2007.03.022

SALGADO, L. H.; BORGES, E. B. D. P. Análise de impacto regulatório: uma abordagem exploratória. Brasília, DF: IPEA, 2010.

SILVA, D. A. V. Monitoramento para avaliação do desempenho regulatório do Inmetro. 2014. Tese (Doutorado) - Instituto Alberto Luiz Coimbra de Pós-Graduação e Pesquisa de Engenharia, Universidade Federal do Rio de Janeiro, Rio de Janeiro, 2014.

SIMÕES, M. G.; SHAW, I. S. Controle e modelagem fuzzy. São Paulo: Edgard Blücher, 2007.

TAKAGI, T.; SUGENO, M. Fuzzy identification of systems and its applications to modeling and control. Systems, Man and Cybernetics, IEEE Transactions on, v. 15, n. 1, p. 116-132, 1985. http://dx.doi.org/10.1109/TSMC.1985.6313399

TURRIONI, J. B.; MELLO, C. H. P. Metodologia de pesquisa em engenharia de produção. Itajubá: Programa de Pós-Graduação em Engenharia de Produção da UNIFEI, 2012.

WANG, L.-X.; MENDEL, J. M. Generating fuzzy rules by learning from examples. IEEE International Symposium on Intelligent Control, p. 263-268, 1991.

http://dx.doi.org/10.1109/21.199466

ZADEH, L. Fuzzy sets. Inf. Control, v. 8, n. 3, p. 338-353, 1965. http://dx.doi.org/10.1016/S00199958(65)90241-X

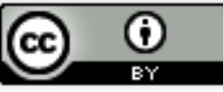

Artigo recebido em 14/07/2014 e aceito para publicação em 17/06/2015.

DOI: http://dx.doi.org/ 10.14488/1676-1901.v15i3.1712 